\title{
El desarrollo de competencias profesionales en el área de Auditoría Informática a través del aprendizaje móvil
}

Otero Escobar, Alma Delia

Universidad Veracruzana, México

aotero@uv.mx

Resumen - El desarrollo de competencias profesionales es una de las finalidades de la educación superior, es por ello que esta investigación propone una aplicación basada en aprendizaje móvil como apoyo para el desarrollo de una auditoría informática en un entorno real, validandose posteriormente la adquisición de competencias de los participantes, la investigación es de corte cuantitativo. Los resultados manifiestan de manera clara la aceptación de la aplicación de aprendizaje móvil y el desarrollo de competencias profesionales entre los participantes.

Palabras clave - Auditoría Informática; Competencias; Aprendizaje móvil;

\begin{abstract}
The development of professional competences is one of the purposes of higher education, which is why this research proposes an application based on mobile learning as support for the development of a computer audit in a real environment, subsequently validating the acquisition of skills of participants, the research is quantitative. The results clearly show the acceptance of the mobile learning application and the development of professional skills among the participants.
\end{abstract}

Keywords - Computer audit; Competencies; Mobile learning;

Interconectando Saberes, 2020

ISSN: 2448-8704

\section{INTRODUCCIÓN}

El aprendizaje móvil se concibe hoy en día como una oportunidad para que el estudiante desarrolle competencias profesionales en diversos ámbitos al ser una herramienta que puede usarse en cualquier lugar y a cualquier hora.

La fácil adquisición y diversidad de dispositivos móviles hace posible que cualquier estudiante universitario tenga acceso al aprendizaje móvil, lo cual se considera una oportunidad para implementar una aplicación basada en aprendizaje móvil que permita a los estudiantes el desarrollo de competencias profesionales al servir como herramienta para la revisión teórica y la aplicación práctica en el desarrollo de una auditoria informática en un entorno real.

Fecha de Recepción: 15 de febrero de 2020

Fecha de Aceptación: 19 de junio de 2020 Fecha de Publicación: 24 de julio de 2020 
GSMA Intelligence predice que, el número de suscriptores móviles alcanzará los 5.900 millones en el año 2025, lo que equivale al $71 \%$ de la población mundial (GSMA, 2019), lo cual es un panorama que facilita la adopción del aprendizaje móvil.

Por su parte StatCounter dedicada al análisis de tráfico Web, desde octubre de 2016 comunicó que el acceso a internet mediante dispositivos móviles supero por primera vez a los equipos de escritorio en todo el mundo (Simpson, 2016).

Para el año 2020, el mercado de los dispositivos móviles ha superado todo el tiempo al de dispositivos de escritorio en más de 4 puntos porcentuales, StatCounter (2020).

El continuo crecimiento del número de conexiones móviles y de la cantidad de suscriptores de servicios móviles en todo el mundo constituye un factor esencial en la introducción de estos dispositivos en la educación y por ende en la evolución y el desarrollo, a escala global, de lo que hoy se conoce como aprendizaje móvil o mlearning. Esto queda reflejado en la literatura en investigaciones como (Moreira, Pereira, Durão, y Ferreira, 2018).

La implementación, el uso y el desarrollo de las tecnologías móviles han transformado el panorama educativo, aportando a la educación no sólo movilidad sino también conectividad, ubicuidad y permanencia, características propias de estos dispositivos. Esto ha traído consigo un cambio de paradigma en el empleo de las TIC en la educación en general. (Valero, Roura, y Sánchez, 2012)

En la Universidad Veracruzana desde el año 2004 se implementó un modelo educativo basado en competencias en busca de una mejor formación y preparación que permita que los estudiantes cuenten con mayores herramientas al enfrentarse a la vida profesional.

En particular en la Licenciatura en Sistemas Computacionales Administrativos existen Experiencias Educativas que promueven la vinculación entre los estudiantes y el sector productivo, entre ellas se encuentra Auditoría Informática cuya unidad de competencia es que el estudiante identifique los conceptos básicos de la auditoría informática para el seguimiento y control de los servicios y consultoría, con la finalidad de aplicarlos a situaciones reales, desarrollando la capacidad y la habilidad para controlar, medir y sopesar los riesgos a los que estar sometidos los procesos y servicios dentro de las organizaciones.

Es así como se tomó la decisión de hacer uso del aprendizaje móvil para llevar a cabo una auditoría informática en un contexto real. 


\section{DEFINICIONES IMPORTANTES}

\section{Aprendizaje}

El aprendizaje es un proceso por el cual un sujeto adquiere habilidades que producen algún cambio en su forma de ser o actuar, a partir de información nueva y conocimiento o experiencias previos. (Otero, Martínez, González, y Gazca, 2015)

\section{Aprendizaje móvil}

Por su parte, el aprendizaje móvil cuenta con múltiples definiciones, se presentan algunas de las más recientes y relevantes para el desarrollo del presente estudio.

De manera general el aprendizaje móvil implica la utilización de las tecnologías móviles al servicio de los procesos asociados con la enseñanza y el aprendizaje. Traxler y Vosloo (2014) se refieren al aprendizaje móvil como la utilización de tecnología móvil, sola o en combinación con cualquier otro tipo de tecnología de la información y las comunicaciones, a fin de facilitar el aprendizaje en cualquier momento y lugar.

Aprendizaje móvil como la utilización de dispositivos móviles mediante la Internet inalámbrica para mejorar la calidad y facilitar el acceso a recursos y servicios, así como lograr el intercambio y colaboración en la sociedad del conocimiento. (Otero et al., 2015)
Santiago, Trabaldo, Kamijo y Fernández (2015) denominan al aprendizaje electrónico móvil como una metodología de enseñanza y aprendizaje valiéndose del uso de pequeños y maniobrables dispositivos móviles, tales como teléfonos móviles, agendas electrónicas, tablets $\mathrm{PC}$, pocket $\mathrm{PC}, \mathrm{i}$ Pods y todo dispositivo de mano que tenga alguna forma de conexión inalámbrica.

Al-Hunaiyyan, Al-Sharhan, y Alhajri (2016) plantean el aprendizaje móvil como una estrategia de aprendizaje que proporciona a los estudiantes las capacidades de aprendizaje del siglo XXI con el fin de que puedan utilizar una amplia gama de los dispositivos informáticos portátiles. Estos dispositivos están conectados a través de la red del aula inteligente $\mathrm{o}$ en Internet a los componentes inteligentes $\mathrm{o}$ de contenido de aprendizaje y recursos educativos interactivos en línea.

De la revisión y análisis de las definiciones anteriores se destacan algunos elementos según el orden en que fueron expuestas las definiciones:

- Procesos de aprendizaje

- Relación con otras tecnologías de la información y las comunicaciones

- Independencia del lugar y el tiempo

- Facilidad de acceso a recursos y servicios educativos en línea

- Metodologías de enseñanza

- Estrategias de aprendizaje

- Aulas inteligentes

- Sistemas de gestión de aprendizaje

- Aprendizaje colaborativo, interactivo e innovador 
Es así como se propone aprovechar el uso del aprendizaje móvil como herramienta en la para el desarrollo de competencias profesionales en el desarrollo de una auditoría en informática.

De acuerdo con Taleb, Ahmadi, y Musavi (2015), se espera que el aprendizaje sea demostrado con resultados, lo que los estudiantes pueden hacer a partir de aquello que saben (conocimiento). Los resultados reflejan habilidades, actitudes y conocimientos teórico-prácticos desarrollados por el profesional, ya que se considera que posee competencia profesional quien dispone de los conocimientos, destrezas y aptitudes necesarios para ejercer una profesión y que puede además resolver problemas profesionales de forma autónoma y flexible, por lo que está capacitado para colaborar en su entorno profesional y en la organización del trabajo. Se entiende que una persona está cualificada cuando en su desempeño laboral obtiene los resultados esperados, con los recursos y el nivel de calidad debido, por tanto, se identifica una oportunidad para demostrar el desarrollo de competencias a través del aprendizaje móvil.

\section{Competencias profesionales}

La UNESCO define competencia como el conjunto de comportamientos socioafectivos y habilidades cognoscitivas, psicológicas, sensoriales y motoras que permiten llevar a cabo adecuadamente un desempeño, una función, una actividad o una tarea.
La competencia por tanto es la capacidad de un profesional para tomar decisiones, con base en los conocimientos, habilidades y actitudes asociadas a la profesión, para solucionar los problemas complejos que se presentan en el campo de su actividad profesional.

El enfoque de la Experiencia Educativa (EE) Auditoría Informática articula conocimientos globales, conocimientos profesionales y experiencias laborales, se propone reconocer las necesidades y problemas de la realidad. Tales necesidades y problemas se definen mediante el diagnóstico de las experiencias de la realidad social, de la práctica de las profesiones, del desarrollo de la disciplina y del mercado laboral. Esta combinación de elementos permite identificar las necesidades hacia las cuales se orientará la formación profesional, de donde se desprenderá también la identificación de las competencias profesionales integrales o genéricas, indispensables para el establecimiento del perfil de egreso del Licenciado en Sistemas Computacionales Administrativos (LSCA).

Se considera como competencia específica para el LSCA la que se busca desarrollar en los estudiantes que cursan la EE de Auditoría en Informática ya que han sido definidas como los atributos que deben adquirir los futuros graduados durante la estancia en la universidad y deben ser definidas por la experiencia propia de los estudiantes además tienen impacto directamente con la formación profesional que deben poseer los futuros egresados. 


\section{Auditoria en informática}

La palabra auditoría proviene de auditor, que es el que tiene virtud de oír, el diccionario lo define como: revisor de cuentas colegiado. El auditor tiene la virtud de oír y revisa cuentas, pero debe estar encaminado a un objetivo específico, que es el evaluar con eficiencia y eficacia con que se está operando para que, por medio del señalamiento de cursos alternativos de acción, se tomen decisiones que permitan corregir los errores, en caso de que existan, o bien mejorar la forma de actuación (García, 2001).

En otras palabras, se dice que auditoría se puede considerar como el examen crítico y sistemático que realiza una persona o grupo de personas independientes del sistema auditado, que puede ser una persona, organización, sistema, proceso, proyecto o producto.

La auditoría en informática se refiere a la revisión práctica que se realiza sobre los recursos informáticos con que cuenta una entidad con el fin de emitir un informe o dictamen sobre la situación en que se desarrollan y se utilizan esos recursos.

También se puede definir como una disciplina que se refiere al análisis de las condiciones de una instalación informática por un auditor externo o interno que realiza un dictamen sobre distintos aspectos informáticos.
Una auditoría en general se caracteriza por ser realizada de forma analítica, sistemática y con amplio sentido crítico por parte del profesional que sea responsable de realizar el examen o evaluación, es decir debe ser objetiva, imparcial, integra, recurrente, selectiva y claramente profesional (Facultad de Ciencias Contables, Económicas y Administrativas, 2003). Así mismo explica que los patrones de cada auditoría dependen según el contexto en que se aplique, es decir qué tipo de auditoría es llevada a cabo.

La Auditoría informática y el aprendizaje móvil en esta investigación llegan a un punto de convergencia en búsqueda del logro de competencias profesionales de los estudiantes ya que se logra la aplicación de una auditoría real.

La Fig. 1 muestra los elementos considerados en la aplicación móvil, tales como plataforma de Moodle Mobile diseñada bajo el modelo 1:1, Valiente(2011), el curso seleccionado de Auditoría Informática, el cual se desglosa en dos secciones: saberes teóricos y saberes prácticos, en donde los saberes prácticos son representados por los instrumentos para la evaluación en una auditoría informática, todo esto es para que el estudiante que cuente con conocimientos previos haga uso de ellos de forma que les sea de utilidad. 
Figura 1. Mapa conceptual de Aprendizaje móvil en la Auditoría Informática

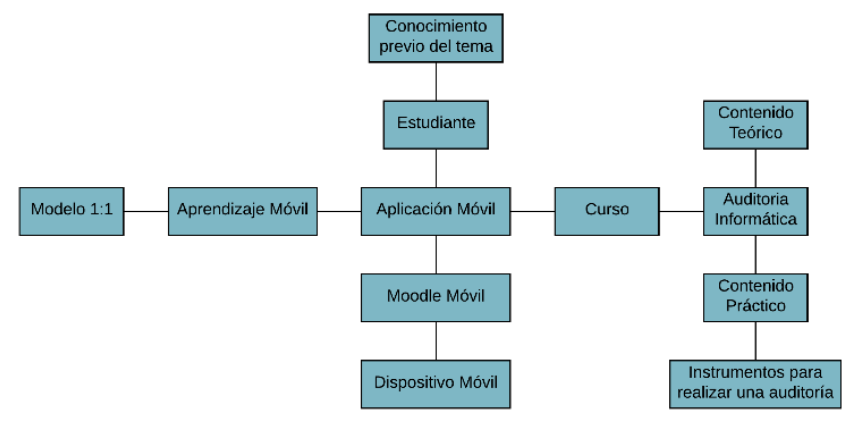

Fuente: Elaboración propia

\section{Metodologías de Auditoría Informática}

Se consideraron diversos autores como Gómez, V. (2014), Lucero, A. (2012) y Hernández, A. (2010) se consultaron para poder incluir los elementos necesarios e indispensables al realizar una auditoría en informática.

Una vez seleccionada la metodología de auditoría en informática se llevó a cabo el diseño, desarrollo e implementación de la aplicación de aprendizaje móvil para llevar a cabo su aplicación en un caso real.

Una vez implementada la aplicación de aprendizaje móvil se subió a un servidor en la nube para fines de estar disponible en cualquier momento $y$ en cualquier lugar para que los estudiantes pudieran hacer uso de ella.

\section{MetOdOLOGíA DE INVESTIGACIÓN}

El objetivo de esta investigación fue promover el desarrollo de competencias profesionales en el área de Auditoría Informática a través del aprendizaje móvil partiendo de la hipótesis de que el aprendizaje móvil como herramienta para realizar la Auditoría Informática coadyuva en el desarrollo de competencias profesionales entre los estudiantes universitarios.

Se trató de una investigación de corte cuantitativo, ya que se caracteriza por obtener respuestas de la población a preguntas específicas. Además de que el método cuantitativo está basado en una investigación empírico-analista. Basa sus estudios en números estadísticos para dar respuesta a unas causas-efectos concretas.

El aspecto empírico-analista se ve representado en la etapa de implementación del aprendizaje móvil ya que a través de esto se obtendrá un análisis de las competencias profesionales adquiridas.

El cuestionario para la medición de las competencias desarrolladas se diseñó exprofeso, con una escala Likert de $2=$ En desacuerdo, $3=\mathrm{Ni}$ de acuerdo ni en desacuerdo, $4=$ De acuerdo y $5=$ Totalmente de acuerdo, solicitando a los participantes expresar su grado de acuerdo o desacuerdo con respecto a cada ítem. 
El cuestionario estuvo compuesto por ítems que medían las competencias adquiridas a través del aprendizaje móvil a la hora de realizar la auditoría informática.

Entre las categorías examinadas se destacan: actividades que permitan la adquisición de competencias; actividades que facilitan la comprensión de los contenidos educativos; las actividades de aprendizaje ayudan a mejorar o fortalecer habilidades para llevar acabo la Auditoría Informática; las actividades permiten a los estudiantes integrar información nueva con aprendizaje previo; las actividades promueven la creatividad permitiendo al estudiante generar sus propias soluciones; las actividades reflejan practicas relevantes a la vida real o profesional al realizar una Auditoría Informática; las herramientas (cuestionarios) son congruentes con las capacidades de los estudiantes (ni muy fáciles, ni muy difíciles); existen actividades o métodos para evaluar el aprendizaje de la Auditoría Informática y, finalmente si las actividades se aprovechan las funciones o ventajas que aportan el uso de los dispositivos móviles (hacer fotos, grabar video o audios, mensajería de textos, etc.).

El muestreo seleccionado para verificar el impacto del aprendizaje móvil en el desarrollo de las competencias de los estudiantes es no probabilístico a conveniencia por así ser requerido en la investigación.
Los criterios de inclusión fueron: ser estudiante de la LSCA, y que se encontraran cursando o que hayan cursado la EE de Auditoría Informática.

Para la selección de los participantes se hizo bajo el criterio de inclusión, cada uno de estos alumnos deberá estar inscrito en la LSCA en el campus Xalapa y estar cursando la experiencia educativa de Auditoría Informática en el periodo FebreroJulio 2019, además de que cuenten con dispositivos móviles como teléfonos celulares 0 tablets, contar con acceso a internet independientemente de la modalidad, además se tomó en cuenta que el grupo tuviera como proyecto final realizar una auditoría informática a una empresa real tal como lo marca el programa de estudios del curso.

Bajo los criterios antes mencionados se seleccionó a los participantes mediante un muestreo no aleatorio, el cual consiste en aquel muestreo que utilizan criterios con un bajo nivel de sistematización que procuran asegurar que la muestra tenga un cierto grado de representatividad.

La sección 601 con 45 integrantes fue la seleccionada para llevar a cabo el estudio del tema a través del aprendizaje móvil.

Al finalizar la Auditoría Informática a través del Aprendizaje Móvil se aplicó la encuesta a los estudiantes seleccionados para identificar la percepción en el uso y las competencias profesionales alcanzadas. 


\section{REsultados}

El $87 \%$ de los encuestados asegura que la aplicación de aprendizaje móvil de auditoría informática contiene actividades que le permiten adquirir nuevos aprendizaje y habilidades.

En cuanto al efecto que tienen las actividades respecto a el contenido del curso, el $91 \%$ de la muestra dio respuestas afirmativas, lo que se traduce en que el contenido practico diseñado fue claro y de ayuda para la comprensión de los saberes teóricos proporcionados previamente a través de la aplicación móvil. Por otro lado, el 91\% de la muestra considero que todas las actividades proporcionadas (los instrumentos y las técnicas incluidas) ayudaron a los alumnos a realizar la auditoría informática.

En cuanto a la integración de información nueva con aprendizajes previos, el 85\% están totalmente de acuerdo con ello sin embargo el otro $15 \%$ se mantiene sin comentarios ni a favor ni en contra lo cual se puede interpretar a que los aspectos prácticos no son proporcionados directamente en su curso presencial.

Se cuestionó si se considera que el uso de la aplicación móvil promueve la creatividad permitiendo al estudiante generar sus propias soluciones dando por resultado que el $82 \%$ afirma que dentro de la aplicación existen actividades que promueven el desarrollo de criterios profesionales para generar soluciones a la auditoría.
Al respecto de si las actividades de la aplicación móvil reflejan prácticas aplicables a la vida real al realizar una auditoría informática solo el 9\% da una respuesta negativa lo que significa que la adaptación de los instrumentos prácticos en las empresas que auditaron no fue idónea.

Los cuestionarios de la aplicación móvil en un $98 \%$ se consideraron de fácil aplicación y comprensión.

Al indagar acerca de los métodos para evaluar el aprendizaje y desarrollo de la competencia profesional de la auditoría informática el $18 \%$ se encontró en el rango intermedio, lo que se puede interpretar como una carencia de un método de evaluación del aprendizaje y es que no fue un método cuantitativo el que se usó, si no uno cualitativo, ya que si llegaba a concluir el curso y hacer uso de la sección de dictamen es que realmente había logrado las metas.

En cuanto al aprovechamiento de los recursos del dispositivo móvil (fotos, grabadora, audios, mensajería, etc.) el $11 \%$ consideró que esto no fue aplicado y esto es debido a que solo se mencionó como una recomendación la cual hacer uso de ellos estaba a criterio personal y un $45 \%$ si considero su uso. 
En cuanto al trabajo colaborativo entre los integrantes del equipo, al hacer uso de la aplicación móvil el 31\% dio una respuesta neutra y se puede interpretar como que dentro de la aplicación no se les dio la iniciativa para realizar actividades conjuntas fuera de las conversaciones o discusiones de algún tema mediante foros o chats.

El $51 \%$ de los estudiantes estuvieron de acuerdo en que la aplicación móvil permite compartir información, fotos, vídeos o documentos relacionados con la auditoría informática.

Finalmente, el $80 \%$ de los encuestados considero la aplicación móvil con un diseño agradable y atractivo.

\section{CONCLUSIONES}

Con base en la investigación realizada se identifican elementos muy significativos, se considera la aplicación de aprendizaje móvil adecuada para lograr las competencias profesionales al realizar una auditoría informática.

Tanto los contenidos, como los cuestionarios de la auditoría informática descritos como actividades en la aplicación móvil fueron aceptados favorablemente.

De acuerdo con respuestas y comentarios de los estudiantes que hicieron uso de la aplicación de aprendizaje móvil algunos pensaron que la plataforma contaba con un diseño muy rustico y con colores no muy agradables, por tanto, la aplicación tuvo diferentes puntos a favor y otros en contra, sin embargo, no fue completamente insatisfactoria para el estudiante debido a que cumplía con lo básico para su función principal.

Algo muy importante respecto a los instrumentos para la auditoria informática que se consideró desde un inicio fue la adaptabilidad en las empresas y en esto se puede decir que se cumplió lo esperado ya que se tomó como enfoque principal las medianas y pequeñas empresas, ya que se caracterizan por no contar con muchos recursos y áreas de aspectos tecnológicos, sin embargo para algunas empresas completamente enfocadas a las tecnologías de la información el uso de los instrumentos proporcionados no fue completamente adaptable, ya que contienen aspectos muy básicos para ellos, a pesar de eso algunos elementos si fueron rescatables. Se puede decir a grandes rasgos que la adaptabilidad del instrumento fue propicia dentro de lo esperado.

Para concluir se destaca que el estudiante al conocer la aplicación móvil mostro interés respecto al uso de una nueva herramienta con la finalidad de agilizar el proceso de evaluación de las empresas con las que se encontraban trabajando, pero para la etapa de implementación y hacer uso de ella realmente pocos tuvieron el espíritu curioso para jugar con el contenido y así familiarizarse con cada una de las opciones y como segundo punto se pudo observar que realmente pocos estudiantes leyeron a profundidad el contenido proporcionado y gran parte fue directamente en búsqueda de las 
instrumentos de evaluación, esto se puede deber a que al ser elementos que se encuentran colocados para su descarga o en uso externo y que de alguna forma eran lo más importante dentro de todo el contenido, los estudiantes no consideraron pertinente leer todo y ver los videos de cada tema.

Se identificó limitantes en la información que se presenta a los estudiantes en la clase tradicional por lo que la aplicación de aprendizaje móvil promueve el desarrollo de las competencias profesionales además de considerarse como un aliado fundamental a la hora realizar la auditoria informática.

Algunas aportaciones de esta investigación fueron: la preparación y configuración de una aplicación basada en aprendizaje móvil para el logro de las competencias profesionales al realizar una auditoria informática; contar con instrumentos para realizar una auditoría informática actualizados y adaptables para diversas áreas de una empresa y se proporcionó una serie de técnicas al estudiante para realizar la auditoría informática de manera adecuada.

\section{REFERENCIAS}

Al-Hunaiyyan, Al-Sharhan, y Alhajri (2016). Perceptions and challenges of mobile learning in Kuwait. Recuperado de https://www.researchgate.net/publication/312 294239 Perceptions and challenges of $\mathrm{m}$ obile learning in Kuwait

Argundin, Y. (2007). Educación basada en competencias, Trillas, México.
Facultad de Ciencias Contables, Económicas y Administrativas. (2003). Características de la auditoria. Obtenido de http://fccea.unicauca.edu.co/old/tgarf/tgarfse $\underline{\text { 3.html }}$

García, J. A. (2001). Auditoria en Informática. McGraw-Hill.

Gómez, L. Antonio, J. (2012). Análisis y gestión de riesgos utilizando la metodología Magerit. Universidad de Cuenca.

Gómez V. (2014). Evaluación de la seguridad de la información con la metodología Octave, Institución Universitaria Pascual Bravo.

GSMA Inteligence (2019). La economía móvil en América Latina. Recuperado de https://www.gsmaintelligence.com/research/ ?file=1861019d0f5f513343ec6c3f87119775\& download

Hernández, A. (2010). Auditoria Informática y Gestión de Tecnología De Información y Comunicación (TICs). Compendium, 13(25), 3-4.

Moreira, F. Santos, C., Durao, N. (2018). A comparative study about mobile learning in Iberian Peninsula Universities: Are professors ready? Recuperado de https://www.researchgate.net/publication/320 348558 A comparative study about mobil e learning in lberian Peninsula Universitie s Are Professors Ready

Otero Escobar, A. D., Martínez Castillo, J., González Benítez, R. Á., y Gazca Herrera, L. A. (2015). OpenEducalML: un modelo de aprendizaje móvil abierto a la Educación Superior. PEARSON.

Simpson, R. (2016). Mobile and tablet internet usage exceeds desktop for first time worldwide. Recuperado de https://gs.statcounter.com/press/mobile-andtablet-internet-usage-exceeds-desktop-forfirst-time-worldwide

Simpson, R. (2020). Desktop vs Mobile vs Tablet Market Share Worldwide. Recuperado de https://gs.statcounter.com/platform-marketshare/desktop-mobile-tablet 
Traxler, J., Vosloo, S. (2014). Introducción: las perspectivas del aprendizaje

móvil. Perspectivas 44,13-28 Recuperado

de https://doi.org/10.1007/s11125-014-9296-

$\underline{z}$

Roegiers, X. (2016). Marco conceptual para la evaluación de competencias. Recuperado de

https://unesdoc.unesco.org/ark:/48223/pf000 $\underline{0245195 \mathrm{spa}}$

Santiago, R., Trabaldo, S., Kamijo, M., y Fernández, Á. (2015). Mobile learning: nuevas realidades en el aula. Digital Text.

Taleb, Z., Ahmadi, A., \& Musavi, M. (2015). The Effect of M-learning on Mathematics Learning. Procedia - Social and Behavioral Sciences, 171, 83-89. Recuperado de https://doi.org/10.1016/j.sbspro.2015.01.092

Valero, C., Roura, M, Palacín, A. (2012). Tendencias actuales en el uso de dispositivos móviles en educación. Recuperado de http://educoas.org/portal/la educacion digita I/147/pdf/ART UNNED EN.pdf

Valiente, O. (2011). Los modelos 1:1 en educación. Prácticas internacionales, evidencia comparada e implicaciones políticas. Número 56 Mayo-Agosto / MaioAgosto 2011. Recuperado de https://rieoei.org/historico/documentos/rie56a $\underline{05 . h t m}$

Verdejo, P. (2006). Modelo para la Educación y Evaluación por Competencias (MECO), México. 